\title{
Structure and Composition Analysis of Core-Shell Structure Bimetallic Concave Nanocubes by Aberration-Corrected Scanning Transmission Electron Microscopy
}

\author{
Ning Lu ${ }^{1}$, Jinguo Wang ${ }^{1}$, Shuifen $\mathrm{Xie}^{2}$, Guannan $\mathrm{He}^{3}$, Younan $\mathrm{Xia}^{2}$, Moon J. Kim ${ }^{1,4}$ \\ 1. Department of Materials Science and Engineering, The University of Texas at Dallas, Richardson, \\ Texas 75080 USA \\ 2. The Wallace H. Coulter Department of Biomedical Engineering, Georgia Institute of Technology and \\ Emory University, School of Chemistry and Biochemistry and School of Chemical and Biomolecular \\ Engineering, Georgia Institute of Technology, Atlanta, GA 30332 USA \\ 3. Department of Chemistry, Washington University, St. Louis, Missouri 63130 USA \\ 4. Department of Nanobio Materials and Electronics, World Class University, Gwangju Institute of \\ Science and Technology, Gwangju 500-712 Korea
}

Bimetallic nanocubes synthesized by seed-mediated growth have the advantage of coupling the catalytic or optical properties of one metal with those of another metal and form multifunctional nanocrystals. Nanocrystals with concave faces have attracted great interest because of the high-index facets on surfaces. Recently, we have studied two kinds of bimetallic concave nanocubes, $\mathrm{Rh}$ on $\mathrm{Pd}$ seeds $(\mathrm{Pd} @ \mathrm{Rh})$ and $\mathrm{Au}$ on $\mathrm{Pd}$ seeds $(\mathrm{Pd} @ \mathrm{Au})$. Aberration $(\mathrm{Cs})$ corrected high angle angular dark field (HAADF) - scanning transmission electron microscopy (STEM) with energy dispersive $\mathrm{x}$-ray spectroscopy (EDS) have been proven to be an unique and effective site-specific analysis tool at atomic and nano scale to investigate the crystal structure and chemistry of nanocrystals. We report here the structure and chemistry of bimetallic concave nanocubes synthesized by controlled kinetics seeded growth. HAADF-STEM, EDS chemical mapping and line scans were performed in a JEOL ARM200F with STEM Cs corrector operated at $200 \mathrm{kV}$.

Figure 1 shows the structural and compositional characterizations of the Pd@Rh core-shell concave nanocubes. Figure 1d and 1e are the HAADF-STEM images of intermediate state of a synthesis. Atomic HAADF-STEM images reveal the continuous lattice fringes from the Pd core to the $\mathrm{Rh}$ or $\mathrm{Au}$ shell, which indicates an epitaxial relationship between the core and shell metals. Interestingly, the side surfaces of the out-extending edges were nearly parallel to the $\{220\}$ planes, as shown in Figure 1c. Distinct atomic steps extending from the corner to the edge suggest that the overgrowth of $\mathrm{Rh}$ or $\mathrm{Au}$ atoms started from the corners of a Pd seed and then extended to the edges, as indicated in Figure 1e.

The atomic numbers of $\mathrm{Pd}$ and $\mathrm{Rh}$ are 46 and 45, respectively. They are very close. Thus, there is no distinct contrast difference between core Pd and shell Rh in the HAADF images, as shown in Figure 1a1e. STEM/EDS mapping and line scans have been used to determine the distributions $\mathrm{Pd}$ and $\mathrm{Rh}$ in the concave nanocube. Figure 1f demonstrates an EDS mapping of Pd@Rh nanocube. STEM/EDS mapping and line scans confirm that the out-extending corners and edges were dominated by Rh or Au, while the cubic core was essentially made of pure Pd. The multiple linear least squares (MLLS) fitting is applied to solve the EDS peak overlap problem in Pd@Rh system by using the standard pure Rh and Pd EDS spectra.

Through Cs-corrected STEM characterization, a site-specific growth mechanism of the bimetallic concave nanocubes has been demonstrated. [1-2]. 


\section{References:}

[1] S. Xie et al., Angewandte Chemie - International Edition 51 (2012) 10266.

[2] G. He et al., ChemCatChem 4 (2012) 1668.

[3] The authors acknowledge funding from the World Class University Program (by MEST through NRF (R31-10026)).
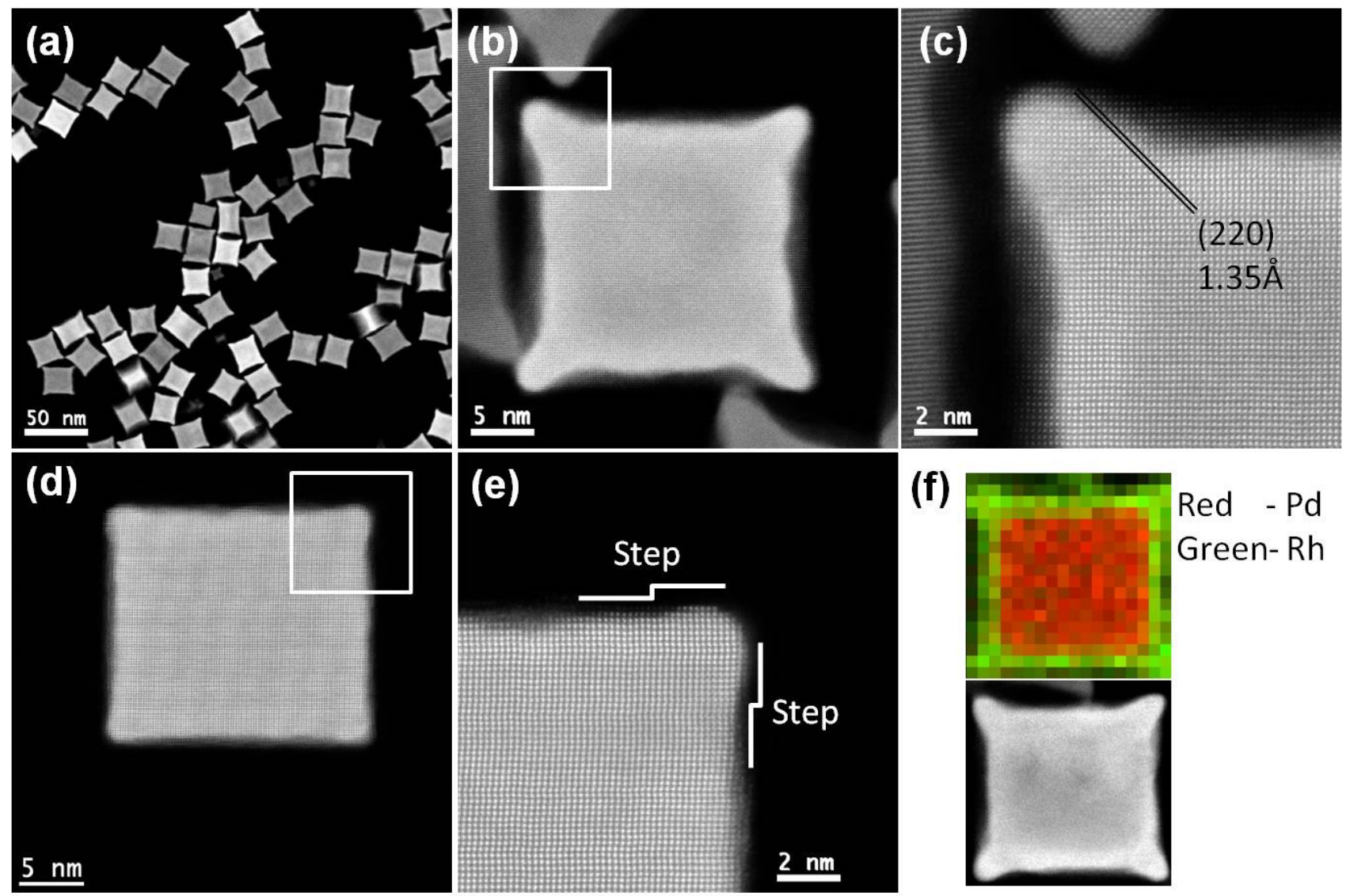

Figure 1. Structural and compositional characterizations of the Pd@Rh core-shell, concave nanocubes. (a,b) HAADF-STEM images; (c) atomic image of the region marked in (b) in $<100>$ zone axis; (d) HAADF-STEM image of intermediate state of a synthesis; (e) atomic image of the region marked in (d) in $<100>$ zone axis; (f) EDS mapping of an individual Pd-Rh nanocube, $\mathrm{Pd}$ as red and $\mathrm{Rh}$ as green. 